\title{
Review Article \\ Small Vessel Cerebrovascular Disease: The Past, Present, and Future
}

\author{
Réza Behrouz, ${ }^{1,2}$ Ali R. Malek, ${ }^{3}$ and Michel T. Torbey ${ }^{1,2}$ \\ ${ }^{1}$ Division of Cerebrovascular Diseases \& Neurocritical Care, Department of Neurology, The Ohio State University College of Medicine, \\ 395 West 12th Avenue, 7th Floor, Suite 766, Columbus, OH 43210, USA \\ ${ }^{2}$ Neurovascular Stroke Center, The Ohio State University Medical Center, 395 West 12th Avenue, 7th Floor, Columbus, \\ OH 43210, USA \\ ${ }^{3}$ Interventional Neurology Program, Comprehensive Stroke Center, St. Mary's Medical Center, 901 45th Street, West Palm Beach, \\ FL 33407, USA
}

Correspondence should be addressed to Réza Behrouz, reza.behrouz@osumc.edu

Received 19 September 2011; Accepted 26 October 2011

Academic Editor: Chelsea S. Kidwell

Copyright (๑) 2012 Réza Behrouz et al. This is an open access article distributed under the Creative Commons Attribution License, which permits unrestricted use, distribution, and reproduction in any medium, provided the original work is properly cited.

\begin{abstract}
Brain infarction due to small vessel cerebrovascular disease (SVCD)—also known as small vessel infarct (SVI) or "lacunar" strokeaccounts for $20 \%$ to $25 \%$ of all ischemic strokes. Historically, SVIs have been associated with a favorable short-term prognosis. However, studies over the years have demonstrated that SVCD/SVI is perhaps a more complex and less benign phenomenon than generally presumed. The currently employed diagnostic and therapeutic strategies are based upon historical and contemporary perceptions of SVCD/SVI. What is discovered in the future will unmask the true countenance of SVCD/SVI and help furnish more accurate prognostication schemes and effective treatments for this condition. This paper is an overview of SVCD/SVI with respect to the discoveries of the past, what is known now, and what will the ongoing investigations evince in the future.
\end{abstract}

\section{Introduction}

A 67-year-old man with long history of hypertension (HTN) presents with a recurrent stroke characterized by acute-onset left hemisensory loss. He suffered his first stroke 8 months ago manifesting as right hemiparesis that resolved over a few weeks. At that time, he underwent an extensive diagnostic workup revealing no evidence of craniocervical large vessel steno-occlusive disease or cardiac abnormalities. A brain magnetic resonance imaging (MRI) was performed at the time of the first stroke and interpreted by the radiologist as an acute "lacunar" infarct within the left internal capsules. $\mathrm{He}$ was started on an aspirin and his antihypertensive medications were "optimized." His MRI now shows a new "lacunar" infarct involving the right thalamus (Figure 1). Dr. Smith, who is the admitting internist, is legitimately concerned and puzzled by this patient's stroke recurrence and consults the neurologist on duty to inquire whether an increase in the aspirin dose or a "switch" to another antiplatelet agent is indicated. In addition, Dr. Smith would also like to know if there is "anything else" that could be done from a diagnostic viewpoint to direct a more effective preventative strategy.

The above is a common clinical scenario encountered by neurologists. Indeed, in the realm of medical decisionmaking, these cases are fundamentally perplexing. It is not simply a conjecture that the patient in the vignette suffers from small vessel cerebrovascular disease (SVCD). As evident by recurrence of his strokes, his condition is probably moderate to severe. He presents with a small vessel infarct (SVI), the typical infarct type associated with SVCD and historically referred to as a "lacunar" stroke. SVIs account for $20 \%$ to $25 \%$ of all ischemic strokes, with an annual incidence of approximately 15 per 100,000 people [1-3]. Over the past few decades, the preponderance of focus with regard to acute and preventative stroke therapy has been placed upon large vessel cerebral ischemia/infarcts. Subsequently, clinical data strictly pertaining to SVI are scarce. Although scientific and technological advancements have empowered cutting-edge care for stroke patients in 


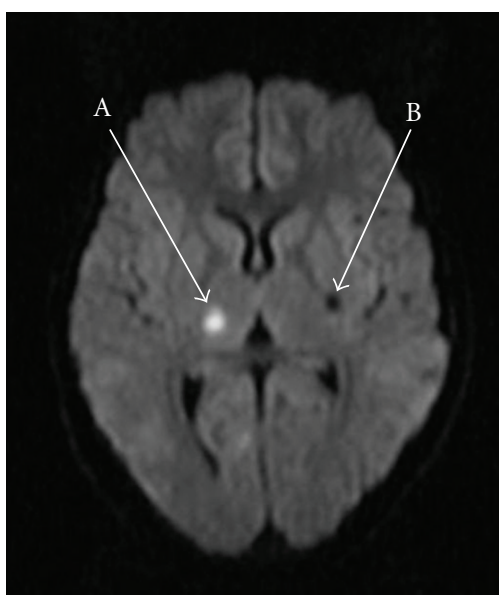

FIGURE 1: Diffusion-weighted imaging (DWI) MRI of the brain showing an acute SVI involving the right thalamus (A) and an older SVI involving the left internal capsule (B).

general, it appears as though management of SVCD remains a perpetual therapeutic conundrum in vascular neurology. In the progressively achieving world of stroke therapeutics, is it fair to boldly suggest that a pathological entity described over 50 years ago remains a remedial destitute? In this paper, we will seek to answer this question by reviewing the past, the present, and the future of SVCD/SVI.

\section{The Past}

A "Lacune" (from Lacuna Latin = lake) is a histopathological nomenclature generated according to what was discovered in postmortem brain autopsies performed in the 1800s. In these patients, small fluid-filled cavities were found in the deep white matter that resembled small lakes, hence the term "lacune" (Figure 2). In the 1960s, Fisher conducted his necropsy series and further acclaimed the term when he described his five classic "lacunar" syndromes: pure motor, pure sensory, ataxic hemiparesis, dysarthria-clumsy hand, and mixed sensorimotor [4]. Fisher observed that these "lacunes" were associated with intrinsic small arteriolar abnormalities that he described as "segmental arteriolar disorganization" [5]. He then assigned the term lipohyalinosis to this form of microangiopathy and histologically detailed the findings as small vessel wall thickening, focal arteriolar dilatation, loss of normal vessel wall architecture, and extravasation of blood components into and through the wall [5]. Based on these findings and the clinical correlations he construed, Fisher certified his conclusions as the "lacunar hypothesis."

Over time, additional mechanisms for SVIs were proposed by Fisher and endorsed by others, including an atherosclerotic pathway with a new appellation: microatheroma [6]. Boiten et al. contended that patients with "lacunar" infarcts can be placed into two groups: those with a single "lacune" who primarily have atherosclerotic risk factors, and those with multiple "lacunes" and a strong history of HTN [7]. In the end, it was proposed that both of these mechanisms are consequences of traditional

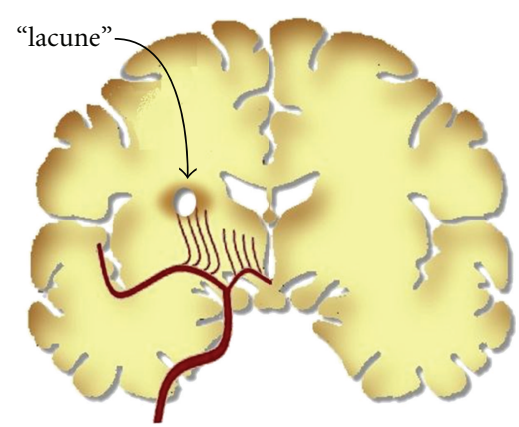

Figure 2: An illustration of coronal cross section of the brain showing a small cavity termed a "lacune" within the subcortical white matter and in the territory of perforating arteries.

vascular risk factors, including and most specifically HTN and diabetes mellitus (DM). Other investigators noticed that not all patients with SVI suffer from HTN and DM. Horowitz et al. reported that in a cohort of 108 patients with SVI, HTN was present in $68 \%$, DM in $37 \%$; both occurred in $28 \%$ and neither occurred in $23 \%$ [8]. They also noticed that of those without HTN or DM, $32 \%$ had a possible carotid or cardiac etiology. A microembolic hypothesis was then advanced suggesting that emboli arising from larger arteries or the heart cause occlusion of the perforating vessels and lead to SVIs [9]. Evidence supporting this hypothesis was in large gathered from studies involving primate models [10].

The definition of a "lacunar" infarct is a lesion that is less than $15 \mathrm{~mm}$ in diameter in the perfusion territory of a small penetrating artery [4]. The terms "lacune," "lacunar infarct," and "lacunar syndrome" have been subject of controversy. Some believe that in a generation where medical eponyms are gradually becoming obsolete, so should outdated terms. Designations should be revised so that they are more descriptive and representative of the pathophysiology of the condition. In this paper, although SVI and "lacune" will be used interchangeably, the former is preferred because it is a fitter depiction of the pathology associated with this stroke subtype.

\section{The Present}

3.1. Disease Behavior. SVCD is not a simplistic entity. When a patient presents with an SVI, it is difficult to determine how extensive his or her SVCD is. If the patient has evidence of extensive white matter disease (chronic microvascular ischemia) or multiple past SVIs on T2/FLAIR MRI, it can be derived that he or she suffers from moderate to severe SVCD and is at risk for recurrent SVIs (Figure 3). Otherwise, an angiographic mechanism to definitively quantify the severity of SVCD does not exist. Subsequently, one cannot ascertain whether the patient's stroke is an isolated event due to a focal segment of small vessel steno-occlusion or representative of a more widespread SVCD. Simply said, only the "tip of the iceberg" may be apparent in our field of view. If this is the case, prognostication and determination of risk of recurrence undeniably become challenging. Over the past few decades, 

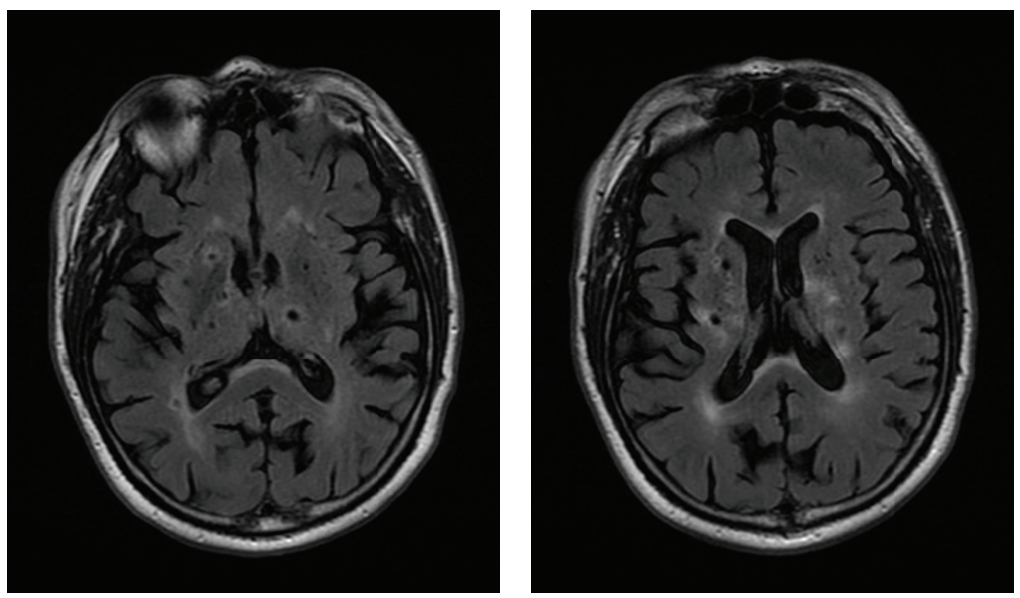

FIgure 3: Fluid Attenuated Inversion Recovery (FLAIR) MRI of the brain showing extensive SVCD evident by multiple, bihemispheric subcortical SVIs.

studies aiming to estimate the rate of SVI recurrence have reported variable figures. One study reported a first-year recurrence rate of $2.83 \%$, while another study looking at 122 patients with a mean time of 58.3 months between the first ever and the recurrent SVI, reported an $83 \%$ rate [11, 12]. A Japanese study, Tottori University Lacunar Infarction Prognosis Study (TULIPS), followed 885 stroke patients for 1 year after the index event and concluded that SVIs were associated with the lowest recurrence rate at 6.2\% [13]. However, in TULIPS, follow-up period was relatively short and it is not clear if all patients received maximal therapy. Regardless of the wavering rates reported in the literature, at least in the long term, SVIs appear to have a tendency to recur. Unfortunately, this is sometimes the case despite optimized stroke preventative therapy.

Soliciting for evidence of prior "silent" or symptomatic SVIs on brain imaging is certainly helpful in predicting the risk of recurrence (Figure 3). A prospective study of 175 patients over a median follow-up period of 12 months found that recurrence rate was $7.7 \%$ in patients with a single SVI and $24.3 \%$ in patients with multiple SVIs on brain imaging [14]. A prior study by De Jong et al. concluded that prognosis for mortality, recurrent stroke, and overall functional outcome in "lacunar" stroke patients with one or more pre-existing silent lesion is less favorable than those without [6]. It is important to bear in mind that a first SVI may or may not be "silent." Therefore, an incipient symptomatic SVI may be the initial glimpse into a more profound yet arcane cerebrovascular disease, even in the absence of preexisting lesions.

Based on ongoing investigations, it appears that a much more complex SVCD/SVI pathophysiology apart from a simple consequence of HTN and DM may be at large. The variable behavior of SVCD/SVI from one patient to another attests to this notion. Although these etiologies may be exclusive of the traditional vascular risk factors, they all culminate in a similar morphology of smallvessel mechanical distortion described by Fisher. Certainly plausible is a genetic association. How is it that within a cohort of patients with the same vascular risk factors, some develop large vessel atherosclerosis while others acquire SVCD with little evidence of large vessel disease? In the Northern Manhattan Stroke Study (NOMASS), the prevalence of HTN, DM, smoking, and hypercholesterolemia did not differ between patients with SVI and those with other stroke subtypes [15]. Is it possible that SVCD is inherited as a distinct genotypic entity similar to CADASIL (cerebral autosomal dominant arteriopathy with subcortical infarcts and leukoencephalopathy) or hereditary forms of cerebral amyloid angiopathy (CAA)?

A recent study showed that family history of stroke might be an independent risk factor for the SVI phenotype with asymptomatic SVIs at a younger age [16]. This study, however, regarded a family history of stroke in a broad sense, not delineating the subtype of the stroke in family members. In an Australian cohort, Jannes et al. reported that polymorphism in the tissue plasminogen activator (tPA) gene $(-7351 \mathrm{C} / \mathrm{T})$ was a risk factor for SVIs, but not other stroke subtypes [17]. Other associations have been reported including polymorphism of the angiotensin converting enzyme (ACE) and interleukin-6 (IL-6) genes $[18,19]$. These studies represent important evidence for a genetic connection and suggest that SVI might result from genetic susceptibility to inflammation-mediated injury in conjunction with atherosclerotic risk factors [20].

Fisher implied in 1969 that "lacunar" infarcts were associated with a relatively good prognosis. Perhaps he anticipated and hoped that novel therapies for SVCD were in the horizon. Considering merely the size of a single, isolated SVI, it may be true that these strokes bear a more favorable prognosis for recovery than other stroke subtypes [21]. However, their natural history may not be as benign as commonly presumed. In a recent study of patients aged 55-85 years with a 12-year follow-up period, the acute index stroke attributable to SVCD was associated with poorer longterm survival and higher risk for cardiac death than other stroke subtypes [22]. The "good prognosis" presumably associated with SVIs is only the case early in the disease course. Years after the infarct, there is an increased risk of death, mainly from cardiovascular causes [23]. Additionally, 
the cumulative effect of recurrent SVIs needs to be considered. As the patient suffers additional SVIs-even in form of "silent" strokes-coalescence and the additive impact of these infarcts may eventually lead to significant physical disability manifested by gait apraxia, urinary incontinence, subcortical vascular dementia, emotional lability/incontinence (pseudobulbar palsy), and affective disorders. SVI caused by SVCD is a major cause of progressive motor deficits [24]. Predictors of mortality after the first-ever SVI are age, degree of neurological dysfunction, and functional disability at day 7 [25]. It is, therefore, imperative that clinicians regard SVCD/SVI as a progressively debilitating condition often characterized by delayed, irreversible consequences.

3.2. Therapeutic Inquest. It has been understood for decades that optimal management of HTN and DM theoretically prevents the progression SVCD and thereby, the recurrence of SVIs. Does this therapeutic endeavor reverse the already progressed disease? What is the "optimal" therapy for secondary SVI prevention beyond HTN and DM control? There has been mounting evidence that SVIs are different than large vessel (or "nonlacunar") infarcts with respect to pathophysiology, etiology, and prognosis. In spite of this, the current American Heart Association's (AHA) Guidelines for the Prevention of Stroke in Patients with Stroke or Transient Ischemic Attack do not differentiate between the two with regard to therapy and preventative measures [26]. Does this "one size fits all" policy apply to SVCD/SVI?

3.2.1. Antiplatelet Agents. The earlier clinical trials looking at the role of antiplatelet agents and anticoagulants clearly demonstrated the benefit of these medications in general secondary prevention of strokes $[27,28]$. These studies did not exclusively look at SVIs, and therefore data specific to this stroke subtype are scarce. For years, the cornerstone of SVI prevention has been control of HTN and DM. Antiplatelet agents are prescribed for SVI patients as a part of a general stroke prevention regimen. However, some neurologists believe that antiplatelet agents are not necessarily of enormous benefit in SVCD and focus should be placed strictly on effectively treating HTN and DM. Because of ethical implications, it is impossible to conduct a clinical trial comparing an antiplatelet agent against placebo in secondary SVI prevention. However, one study did explore that and reported intriguing results. The Cilostazol Stroke Prevention Study (CSPS) was a multicenter, randomized, placebo-controlled, double-blind clinical trial examining the effects of cilostazol on the recurrence of cerebral infarction [29]. Cilostazol is an antiplatelet agent that increases the cyclic adenosine monophosphate levels in platelets via inhibition of phosphodiesterase. Cilostazol was found to reduce the risk of secondary stroke by $41.7 \%$ compared with placebo. CSPS investigators reported that the greatest risk reduction was found in patients who initially had a "lacunar" infarction (43.4\% in cilostazol versus placebo, $P=$ 0.0373 ), suggesting that cilostazol has a specific effect against SVCD. However, it is important to allude to the fact that close to $75 \%$ of patients in each arm had an SVI as the initial event. This unequal distribution of patient population could have created a bias with respect to the results, yet it demonstrated clearly that antiplatelet agents are not futile in secondary prevention of SVIs. A subsequent CSPS-2 study demonstrated the noninferiority of cilostazol to aspirin, but the focus was general stroke prevention and not specific to SVIs [30]. In conclusion, antiplatelet agents are indicated in SVCD for prevention of SVIs. The reason why a patient has a subsequent SVI on an antiplatelet agent is possibly due to the recurrent nature of SVIs and not necessarily a consequence of medication failure.

3.2.2. Statins. The AHA Guidelines also do not address the utility of 3-hydroxy-3-methyl-glutaryl-CoA reductase inhibitors (HMG-CoA RI) or "statins" specifically in secondary prevention of SVIs. As for general stroke prevention, the Guidelines recommend "intensive lipid-lowering" to reduce risk of stroke and cardiovascular events among patients with ischemic stroke or transient ischemic attack (TIA) who have evidence of atherosclerosis or a low-density lipoprotein C (LDL-C) level $\geq 100 \mathrm{mg} / \mathrm{dL}$ [26]. Although the use of statins in treatment of patients with cerebrovascular disease was initially approved by the Food and Drug Administration (FDA) in the wake of the Heart Protection Study, this particular recommendation is largely based on the Stroke Prevention by Aggressive Cholesterol Lowering (SPARCL) study $[31,32]$. SPARCL showed a five-year absolute risk reduction of $2.2 \%(P=0.03)$ for recurrent strokes with administration of high-dose $(80 \mathrm{mg})$ atorvastatin as compared with placebo. Patients with SVI as entry event were included in the study. With a number-needed-to-treat (NNT) of 258 to prevent a first recurrent stroke over one year, many question the ostentatious results of SPARCL. Furthermore, there exists a degree of hesitance among some neurologists to treat SVCD patients with high-dose statins because of risk of intracerebral hemorrhage (ICH). There is some evidence suggesting that patients with SVCD may suffer chronic "silent" cerebral microhemorrhages that could potentially convert to larger hemorrhagic foci or frank ICH [33]. Nonetheless, SPARCL investigators contended that subjects with SVI at baseline who were treated with highdose statins had a benefit in the primary endpoint (combined risk of any fatal or nonfatal stroke) that was almost identical to the benefit in the overall study population, with no overall treatment-related difference in the frequency of fatal $\mathrm{ICH}$ [34]. Indeed, there was an increased rate of $\mathrm{ICH}$ in patients with SVCD and SVI at entry who underwent intensive treatment with statins (HR, 4.99; 95\% CI, 1.71 to 14.61) [34]. However, the authors maintained that the data sustaining this finding lacked statistical validity because of a high tendency towards false positives [34]. Another study of 94 SVI patients treated with $80 \mathrm{mg}$ of atorvastatin per day did not improve severe cerebral vasoreactivity and microvascular endothelial dysfunction assessed by ultrasonographic technology [35]. Given these findings, implementation of statins at high doses in SVCD/SVI patients remains controversial. It is yet to be determined whether the overall benefit of highdose statins in SVCD/SVI patients outweighs the relatively 
higher risk of ICH. Perhaps higher prevalence of HTN in patient with SVCD/SVI plays a contributing role to this risk.

3.2.3. Intravenous Thrombolytics. The National Institutes of Neurological Disorders and Stroke (NINDS) thrombolytic trial reported that all stroke subtypes, including SVIs, responded favorably to intravenous (IV) recombinant tissue plasminogen activator (rtPA) [36]. Some experts argue against this notion. In his textbook entitled Stroke, Caplan expresses his skepticism regarding the benefit of IV rtPA in SVIs. He raises several pitfalls in the NINDS rtPA trial including, less-than-thorough examinations by the investigators, inadequate brain imaging, and dissimilar subject numbers between the treatment and the placebo groups [37]. Caplan further asserts that a committee designated to conduct post hoc analysis of the NINDS rtPA data later suggested that the trial's conclusions pertaining to stroke subtypes were invalid [38]. Until further data is available, there is no evidence suggesting that IV rtPA is of dubious benefit in acute SVI. Therefore, IV rtPA should not be withheld based upon anecdotal declarations, since it remains the only acute therapeutic option in acute SVI. Additionally, It is not always possible to clinically link a "lacunar syndrome," such as pure motor paresis, to an SVI. A non-SVI mechanism is responsible for $25 \%$ of cases that present with a "lacunar syndrome." [15]. If we decide to withhold administration of IV rtPA purely based on clinical grounds (lacunar hypothesis), we could theoretically deprive of treatment 1 out of every 4 patients presenting with a socalled "lacunar syndrome."

3.2.4. Carotid Surgery. One challenging scenario is when one encounters a patient with an SVI and an ipsilateral internal carotid artery (ICA) stenosis. Would this be considered a "symptomatic" ICA stenosis and should this patient undergo carotid endarterectomy (CEA)? Recall that animal models have corroborated the embolic theory for occurrence of SVIs in the face of an ipsilateral ICA disease [10]. In a subgroup analysis, the North American Symptomatic Carotid Endarterectomy Trial (NASCET) group reported that SVIs occurred more often with milder $(<50 \%)$ ICA stenosis, and for patients with $50 \%$ to $99 \%$ stenosis, the relative risk reductions in stroke from CEA were $35 \%$ and $61 \%$ for probable SVI and non-SVI, respectively [39]. The European Carotid Surgery Trialists (ECST) also found that SVIs were associated with milder cases of ICA stenosis, but maintained that small numbers did not allow conclusive evidence on the efficacy of CEA in SVI patients with severe stenosis [40]. The ECST investigators, therefore, concluded that severe ICA stenosis in SVI patients is usually incidental, and so should be deemed "asymptomatic." Tejada et al. found significant incidence of isolated ipsilateral ICA stenosis in patients with SVIs in the ICA territory, particularly ipsilateral multiple SVIs [41]. The authors suggested a significant relationship between the pattern of distribution of SVIs in only one hemisphere and ipsilateral ICA stenosis more than 70\% (OR, 4.4; 95\% CI, 0.9 to $19 ; P=0.03$ ). Unfortunately, the evidence supporting one approach over the other is lacking due to nebulous, conflicting information. Cases could range in complexity from multiple SVIs in the territory of a severely stenotic ICA (and a paucity of SVIs within other territories) to a stenosis in the $50 \%$ to $69 \%$ range and a solitary SVI within its perfusion domain. There is some data suggesting that in patients with moderate ICA stenosis, distinctive ultrasonographic findings (intima-media thickness and cerebrovascular reactivity to hypercapnea) could assist in differentiating between an SVI due to ICA disease and otherwise [42]. Further studies are certainly required to clarify this. Ultimately, careful workup and decision-making needs to take place on a case-bycase basis, bearing in mind individual factors such as age, comorbid conditions, and of course, institutional rates of perioperative complications.

\section{The Future}

The future of SVCD/SVI is contingent upon two main aspects: accurate prognostication and effective management. Autopsy-centered investigations such as those conducted by Fisher are not easy to carry out because of the low case fatality of SVIs [43]. Moreover, histopathological data obtained from these investigations may not provide any additional information with regard to pathophysiology and natural history of the disease. This is unless the intention is to utilize sophisticated staining techniques to discover accumulation within the vessel wall of pathological polypeptides similar to amyloid in CAA or Notch-3 protein in CADASIL.

Prognostication of SVCD/SVI requires the ability to predict the risk of recurrence. If one discounts the unequal follow-up durations between studies, the variable reported recurrence rates could be attributed to a few possible theories. First, multiple etiologic and pathophysiological mechanisms may be implicated in the development of SVCD, all inherently associated with occurrence of SVIs. Again, these mechanisms may be completely exclusive of HTN and DM, or emboldened by them. Explorative endeavors should focus on biological and inflammatory markers that may be more prevalent in patients with SVCD/SVI and potentially amenable to modification and/or therapy. As an example, a recent study determined that in patients with more than one small vessel lesion or extensive white matter disease on brain MRI, there were higher levels of serum tPA and lower levels of plasminogen activator inhibitor type 1 (PAI-1) when compared with patients with a solitary SVI [44]. The authors postulated that differences in activity of components of the fibrinolytic system might be involved in the pathophysiology of SVCD. There is also evidence that implicates chronic endothelial dysfunction assessed by measuring circulating levels of intercellular adhesion molecule 1 (ICAM-1), thrombomodulin (TM), tissue factor (TF), and tissue factor pathway inhibitor (TFPI) [45]. Another study showed that, after adjustment for potential confounders, serum tumor necrosis factor-alpha (TNFalpha) $>14 \mathrm{pg} / \mathrm{mL}$ and ICAM- $1>208 \mathrm{pg} / \mathrm{mL}$ were independently associated with early neurological deterioration and poor outcome at 3 months in patients with SVI [46]. Also, monocyte count might be an inflammatory risk marker 
for development of SVIs [47]. The Levels of Inflammatory Markers in the Treatment of Stroke (LIMITS) in an ongoing prospective, observational study aimed to determine if serum levels of inflammatory markers-such as high-sensitivity creactive protein (hsCRP), serum amyloid A (SAA), CD40 ligand $(\mathrm{CD} 40 \mathrm{~L})$, and monocyte chemoattractant protein1 (MCP-1)—predict recurrent stroke and other vascular events among people with a history of SVI [48]. These biological and inflammatory markers could serve as potential therapeutic targets in the future.

Second, SVCD may be a unique genetic disorder or set of disorders with variable penetrance or expressivity and subsequently with diverse phenotypic severity. Several distinct genes could be involved in the pathophysiology of SVCD. Individual genetic factors may perhaps predispose to specific stroke subtypes only, and not to strokes overall [49]. Exploring these concepts requires long-term, longitudinal investigation of patient pedigrees with specification of stroke subtypes in family members. The Siblings with Ischemic Stroke Study (SWISS) is a multicenter, observational study of sibling pairs and is determined to identify regions of the human genome that correlate with risk of developing ischemic stroke [50]. This study conducts genome-wide screen using DNA obtained from concordant and discordant sibling pairs. Although the SWISS investigators are not conducting subtype analysis (yet), subjects with SVI are included. Once completed, future assays of SWISS could provide an insight into the genetics of SVIs.

Third, the initial SVI may be due to either a focal, isolated small vessel stenosis or a reflection of a more capacious disease. As previously mentioned, angiography is of limited use with respect to precisely gauging the severity of SVCD. However, brain perfusion studies could be useful in that regard. Computed tomographic perfusion- (CTP-) derived subcortical white matter cerebral blood flow (CBF) has been shown to be independently associated with white matter disease severity [51]. It is known that greater white matter disease burden is associated with SVI compared with other ischemic stroke subtypes and therefore a marker of more severe SVCD [52]. But does an MRI devoid of extensive white matter disease completely rule out severe SVCD? Comparison of postmortem MRI and histopathology suggests the existence of "MRI invisible" SVCD [53]. Quantitative MRI techniques, such as diffusion tensor imaging, may help unmask SVCD otherwise undetectable on conventional MRI [53]. Although such sophisticated imaging modalities may not be widely available, prospective research utilizing this technology could provide additional angles for accurate prognostication of SVCD in the years to come.

All of the possibilities mentioned above influence our ability to proximately predict prognosis. They also contribute to the prospect of finding more effective therapies for SVCD/SVI. Closer acumen into the causes of SVCD in advance of HTN and DM can help direct etiologytailored treatments. However, until novel therapeutic options emerge, neurologists are limited to only the medical remedies that are available at present. Hence, the initial step in the therapeutic inquest for SVCD/SVI is optimization of current therapies. There is a paucity of data with regard to optimal blood pressure, serum glucose, and choice of medications in secondary prevention of SVIs. In Prevention Regimen For Effectively avoiding Second Strokes (PRoFESS) where more than $50 \%$ of the patients had suffered an SVI as the index event, there was no difference between clopidogrel and aspirin/extended-release dipyridamole with regard to effectiveness in prevention of recurrent strokes [54]. In another arm of PRoFESS, it was demonstrated that treatment with telmisartan (an angiotensin receptor blocking agent) initiated soon after an ischemic stroke and continued for 2.5 years did not significantly lower the rate of recurrent stroke [55]. The Study of Prevention of Small Subcortical Strokes (SPS3) is an investigational effort seeking to address the optimal therapy for patients with SVI and is expected completion in spring of 2012 [56]. In SPS3, eligible SVI patients are randomized in a double-blind fashion to receive either aspirin $325 \mathrm{mg} / \mathrm{day}$ or a combination of aspirin $325 \mathrm{mg} /$ day and clopidogrel $75 \mathrm{mg} /$ day. Hypertensive SVI patients are randomized to receive antihypertensive therapy with a target systolic blood pressure of either $<150 \mathrm{mmHg}$ or $<130 \mathrm{mmHg}$. Primary outcomes measures are recurrent stroke, major vascular events, or cognitive decline. ICH, systemic hemorrhage, and side effects of aggressive antihypertensive therapy are designated as secondary outcome measures. LIMITS and SPS3 employ the same pool of subjects to determine if inflammatory markers predict which people will respond best to dual antiplatelet therapy (clopidogrel and aspirin). As recommended by the study's Data and Safety Monitoring Board, the National Institute of Neurological Disorders and Stroke (NINDS) stopped the antiplatelet component of the study due to an increased rate of major hemorrhage and death in patients receiving combination antiplatelet therapy. Moreover, a futility analysis demonstrated little likelihood of benefit in favor of aspirin plus clopidogrel on recurrent stroke should the study continue to conclusion. The blood pressure intervention component of the study was allowed to continue.

Other investigative approaches to define more precise therapies could involve comparing various antihypertensive or antihyperglycemic medications in an aim to determine the ideal class of agents for SVI prevention. In closure, experimental therapeutic studies involving patients with SVCD/SVI are much needed.

\section{Conclusion}

The issues raised above encourage taking a broader view in diagnosis and management of SVCD/SVI. Many of the points discussed have been widely known for years and continue to challenge the minds of neurologists and clinicians alike. It is gradually becoming apparent that SVCD/SVI is a much more complex phenomenon than what was described by Fisher decades ago. The "optimal" treatment with regard to prevention of secondary SVIs is still at its minimum. Simply suggesting maximal control of HTN and DM as the ideal therapy and switching back-and-forth between antiplatelet agents are becoming self-fulfilling strategies. Perhaps the 
time has arrived that we diligently look beyond Fisher's "lacunar hypothesis" and seek further explanations as to why SVCD so peculiarly behaves the way it does.

\section{Conflict of Interests}

The authors report no conflict of interests.

\section{References}

[1] H. P. Adams Jr., B. H. Bendixen, L. J. Kappelle et al., "Classification of subtype of acute ischemic stroke: definitions for use in a multicenter clinical trial," Stroke, vol. 24, no. 1, pp. 35-41, 1993.

[2] C. M. Fisher, "Lacunar strokes and infarcts: a review," Cerebrovascular Diseases, vol. 1, pp. 311-320, 1991.

[3] S. Sacco, C. Marini, R. Totaro, T. Russo, D. Cerone, and A. Carolei, "A population-based study of the incidence and prognosis of lacunar stroke," Neurology, vol. 66, no. 9, pp. 1335-1338, 2006.

[4] C. M. Fisher, "Lacunar strokes and infarcts: a review," Neurology, vol. 32, no. 8, pp. 871-876, 1982.

[5] C. M. Fisher, “The arterial lesions underlying lacunes," Acta Neuropathologica, vol. 12, no. 1, pp. 1-15, 1969.

[6] G. De Jong, F. Kessels, and J. Lodder, "Two types of lacunar infarcts: further arguments from a study on prognosis," Stroke, vol. 33, no. 8, pp. 2072-2076, 2002.

[7] J. Boiten, J. Lodder, and F. Kessels, "Two clinically distinct lacunar infarct entities? A hypothesis," Stroke, vol. 24, no. 5, pp. 652-656, 1993.

[8] D. R. Horowitz, S. Tuhrim, J. M. Weinberger, and S. H. Rudolph, "Mechanisms in lacunar infarction," Stroke, vol. 23, no. 3, pp. 325-327, 1992.

[9] N. Futrell, "Lacumar infarction: embolism is the key," Stroke, vol. 35, no. 7, pp. 1778-1779, 2004.

[10] R. L. Macdonald, A. Kowalczuk, and L. Johns, "Emboli enter penetrating arteries of monkey brain in relation to their size," Stroke, vol. 26, no. 7, pp. 1247-1251, 1995.

[11] R. L. Sacco, S. Prabhakaran, J. L. P. Thompson et al., "Comparison of warfarin versus aspirin for the prevention of recurrent stroke or death: subgroup analyses from the warfarin-aspirin recurrent stroke study," Cerebrovascular Diseases, vol. 22, no. 1, pp. 4-12, 2006.

[12] A. Arboix, A. Font, C. Garro, L. García-Eroles, E. Comes, and J. Massons, "Recurrent lacunar infarction following a previous lacunar stroke: a clinical study of 122 patients," Journal of Neurology, Neurosurgery and Psychiatry, vol. 78, no. 12, pp. 1392-1394, 2007.

[13] T. Soda, H. Nakayasu, M. Maeda et al., "Stroke recurrence within the first year following cerebral infarction-Tottori University Lacunar Infarction Prognosis Study (TULIPS)," Acta Neurologica Scandinavica, vol. 110, no. 6, pp. 343-349, 2004.

[14] A. Arauz, L. Murillo, C. Cantú, F. Barinagarrementeria, and J. Higuera, "Prospective study of single and multiple lacunar infarcts using magnetic resonance imaging: risk factors, recurrence, and outcome in 175 consecutive cases," Stroke, vol. 34, no. 10, pp. 2453-2458, 2003.

[15] R. Gan, R. L. Sacco, D. E. Kargman, J. K. Roberts, B. BodenAlbala, and Q. Gu, "Testing the validity of the lacunar hypothesis: the northern Manhattan stroke study experience," Neurology, vol. 48, no. 5, pp. 1204-1211, 1997.
[16] I. L. H. Knottnerus, M. Gielen, J. Lodder et al., "Family history of stroke is an independent risk factor for lacunar stroke subtype with asymptomatic lacunar infarcts at younger ages," Stroke, vol. 42, no. 5, pp. 1196-1200, 2011.

[17] J. Jannes, M. A. Hamilton-Bruce, L. Pilotto et al., "Tissue plasminogen activator $-7351 \mathrm{C} / \mathrm{T}$ enhancer polymorphism is a risk factor for lacunar stroke," Stroke, vol. 35, no. 5, pp. 10901094, 2004.

[18] P. Pullicino, P. L. Kwen, S. Greenberg, A. L. Becker, and N. Glenister, "Angiotensin-converting enzyme gene and lacunar stroke," Stroke, vol. 27, no. 3, pp. 569-570, 1996.

[19] A. Chamorro, M. Revilla, V. Obach, M. Vargas, and A. M. Planas, "The $-174 \mathrm{G} / \mathrm{C}$ polymorphism of the interleukin 6 gene is a hallmark of lacunar stroke and not other ischemic stroke phenotypes," Cerebrovascular Diseases, vol. 19, no. 2, pp. 9195, 2005.

[20] M. Revilla, V. Obach, Á. Cervera, A. Dávalos, J. Castillo, and Á. Chamorro, "A -174G/C polymorphism of the interleukin-6 gene in patients with lacunar infarction," Neuroscience Letters, vol. 324, no. 1, pp. 29-32, 2002.

[21] I. Clavier, M. Hommel, G. Besson, B. Noèlle, and J. E. F. Perret, "Long-term prognosis of symptomatic lacunar infarcts: a hospital-based study," Stroke, vol. 25, no. 10, pp. 2005-2009, 1994.

[22] S. Melkas, J. Putaala, N. K. Oksala et al., "Small-vessel disease relates to poor poststroke survival in a 12-year follow-up," Neurology, vol. 76, no. 8, pp. 734-739, 2011.

[23] B. Norrving, "Long-term prognosis after lacunar infarction," Lancet Neurology, vol. 2, no. 4, pp. 238-245, 2003.

[24] W. Steinke and S. C. Ley, "Lacunar stroke is the major cause of progressive motor deficits," Stroke, vol. 33, no. 6, pp. 15101516, 2002.

[25] A. V. Salgado, J. M. Ferro, and A. Gouveia-Oliveira, "Longterm prognosis of first-ever lacunar strokes: a hospital-based study," Stroke, vol. 27, no. 4, pp. 661-666, 1996.

[26] K. L. Furie, S. E. Kasner, R. J. Adams et al., "Guidelines for the prevention of stroke in patients with stroke or transient ischemic attack: a guideline for healthcare professionals from the american heart association/american stroke association," Stroke, vol. 42, pp. 227-276, 2011.

[27] Z. Chen, P. Sandercock, H. Pan et al., "Indications for early aspirin use in acute ischemic stroke: a combined analysis of 40 000 randomized patients from the Chinese Acute Stroke Trial and the International Stroke Trial," Stroke, vol. 31, no. 6, pp. 1240-1249, 2000.

[28] J. P. Mohr, J. L. P. Thompson, R. M. Lazar et al., "A comparison of warfarin and aspirin for the prevention of recurrent ischemic stroke," New England Journal of Medicine, vol. 345, no. 20, pp. 1444-1451, 2001.

[29] F. Gotoh, H. Tohgi, S. Hirai et al., "Cilostazol stroke prevention study: a placebo-controlled double-blind trial for secondary prevention of cerebral infarction," Journal of Stroke and Cerebrovascular Diseases, vol. 9, no. 4, pp. 147-157, 2000.

[30] Y. Shinohara, Y. Katayama, S. Uchiyama et al., "Cilostazol for prevention of secondary stroke (CSPS 2): an aspirincontrolled, double-blind, randomised non-inferiority trial," The Lancet Neurology, vol. 9, no. 10, pp. 959-968, 2010.

[31] R. Collins, J. Armitage, S. Parish, P. Sleight, and R. Peto, "Effects of cholesterol-lowering with simvastatin on stroke and other major vascular events in 20536 people with cerebrovascular disease or other high-risk conditions," The Lancet, vol. 363, no. 9411, pp. 757-767, 2004.

[32] P. Amarenco, J. Bogousslavsky, A. Callahan et al., "Highdose atorvastatin after stroke of transient ischemic attack. The 
stroke prevention by aggressive reduction in cholesterol levels study (SPARCL)," New England Journal of Medicine, vol. 355, pp. 549-559, 2006.

[33] Y. H. Fan, V. C. T. Mok, W. W. M. Lam, A. C. F. Hui, and K. S. Wong, "Cerebral microbleeds and white matter changes in patients hospitalized with lacunar infarcts," Journal of Neurology, vol. 251, no. 5, pp. 537-541, 2004.

[34] L. B. Goldstein, P. Amarenco, M. Szarek et al., "Hemorrhagic stroke in the Stroke Prevention by Aggressive Reduction in Cholesterol Levels study," Neurology, vol. 70, no. 24, pp. 23642370, 2008.

[35] P. C. Lavallée, J. Labreuche, F. Gongora-Rivera et al., "Placebocontrolled trial of high-dose atorvastatin in patients with severe cerebral small vessel disease," Stroke, vol. 40, no. 5, pp. 1721-1728, 2009.

[36] National Institute of Neurological Disorders and Stroke rtPAStroke Study Group, "Tissue plasminogen activator for acute ischemic stroke," New England Journal of Medicine, vol. 333, no. 24, pp. 1581-1587, 1995.

[37] L. R. Caplan, "Penetrating and branch artery disease," in Stroke: A Clinical Approach, L. R. Caplan, Ed., pp. 291-315, Saunders, Philadelphia, Pa, USA, 4th edition, 2009.

[38] T. J. Ingall, W. M. O'Fallon, K. Asplund et al., "Findings from the reanalysis of the NINDS tissue plasminogen activator for acute ischemic stroke treatment trial," Stroke, vol. 35, no. 10, pp. 2418-2424, 2004.

[39] D. Inzitari, M. Eliasziw, B. L. Sharpe, A. J. Fox, and H. J. Barnett, "Risk factors and outcome of patients with carotid artery stenosis presenting with lacunar stroke," Neurology, vol. 54, no. 3, pp. 660-666, 2000.

[40] J. Boiten, P. M. Rothwell, J. Slattery, and C. P. Warlow, "Ischaemic lacunar stroke in the European Carotid Surgery Trial. Risk factors, distribution of carotid stenosis, effect of surgery and type of recurrent stroke," Cerebrovascular Diseases, vol. 6, no. 5, pp. 281-287, 1996.

[41] J. Tejada, E. Díez-Tejedor, L. Hernández-Echebarría, and O. Balboa, "Does a relationship exist between carotid stenosis and lacunar infarction?" Stroke, vol. 34, no. 6, pp. 1404-1409, 2003.

[42] M. Silvestrini, P. Pasqualetti, R. Baruffaldi et al., "Cerebrovascular reactivity and cognitive decline in patients with Alzheimer disease," Stroke, vol. 37, no. 4, pp. 1010-1015, 2006.

[43] C. Jackson and C. Sudlow, "Comparing risks of death and recurrent vascular events between lacunar and non-lacunar infarction," Brain, vol. 128, no. 11, pp. 2507-2517, 2005.

[44] I. L. H. Knottnerus, J. W. P. Govers-Riemslag, K. Hamulyak et al., "Endothelial activation in lacunar stroke subtypes," Stroke, vol. 41, no. 8, pp. 1617-1622, 2010.

[45] A. Hassan, B. J. Hunt, M. O'Sullivan et al., "Markers of endothelial dysfunction in lacunar infarction and ischaemic leukoaraiosis," Brain, vol. 126, no. 2, pp. 424-432, 2003.

[46] M. Castellanos, J. Castillo, M. M. García et al., "Inflammationmediated damage in progressing lacunar infarctions a potential therapeutic target," Stroke, vol. 33, no. 4, pp. 982-987, 2002.

[47] M. G. Cortina, A. R. Campello, J. J. Conde et al., "Monocyte count is an underlying marker of lacunar subtype of hypertensive small vessel disease," European Journal of Neurology, vol. 15, no. 7, pp. 671-676, 2008.

[48] M. S. V. Elkind, J. M. Luna, C. S. Coffey et al., "The levels of inflammatory markers in the treatment of stroke study (limits): inflammatory biomarkers as risk predictors after lacunar stroke," International Journal of Stroke, vol. 5, no. 2, pp. 117-125, 2010.
[49] P. Jerrard-Dunne, G. Cloud, A. Hassan, and H. S. Markus, "Evaluating the genetic component of ischemic stroke subtypes: a family history study," Stroke, vol. 34, no. 6, pp. 13641369, 2003.

[50] J. F. Meschia, B. M. Kissela, T. G. Brott et al., "The Siblings With Ischemic Stroke Study (SWISS): a progress report," Clinical Medicine and Research, vol. 4, no. 1, pp. 12-21, 2006.

[51] T. J. Huynh, B. Murphy, J. A. Pettersen et al., "CT perfusion quantification of small-vessel ischemic severity," American Journal of Neuroradiology, vol. 29, no. 10, pp. 1831-1836, 2008.

[52] N. S. Rost, R. M. Rahman, A. Biffi et al., "White matter hyperintensity volume is increased in small vessel stroke subtypes," Neurology, vol. 75, no. 19, pp. 1670-1677, 2010.

[53] A. A. Gouw, A. Seewann, W. M. Van Der Flier et al., "Heterogeneity of small vessel disease: a systematic review of MRI and histopathology correlations," Journal of Neurology, Neurosurgery and Psychiatry, vol. 82, no. 2, pp. 126-135, 2011.

[54] R. L. Sacco, H. C. Diener, S. Yusuf et al., "Aspirin and extended-release dipyridamole versus clopidogrel for recurrent stroke," New England Journal of Medicine, vol. 359, no. 12, pp. 1238-1251, 2008.

[55] S. Yusuf, H. C. Diener, R. L. Sacco et al., "Telmisartan to prevent recurrent stroke and cardiovascular events," New England Journal of Medicine, vol. 359, no. 12, pp. 1225-1237, 2008.

[56] O. R. Benavente, C. L. White, L. Pearce et al., "The Secondary Prevention of Small Subcortical Strokes (SPS3) study," International Journal of Stroke, vol. 6, no. 2, pp. 164-175, 2011. 


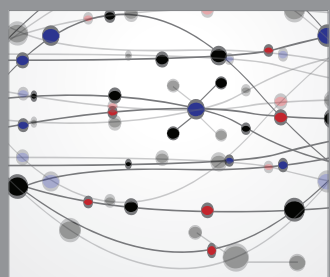

The Scientific World Journal
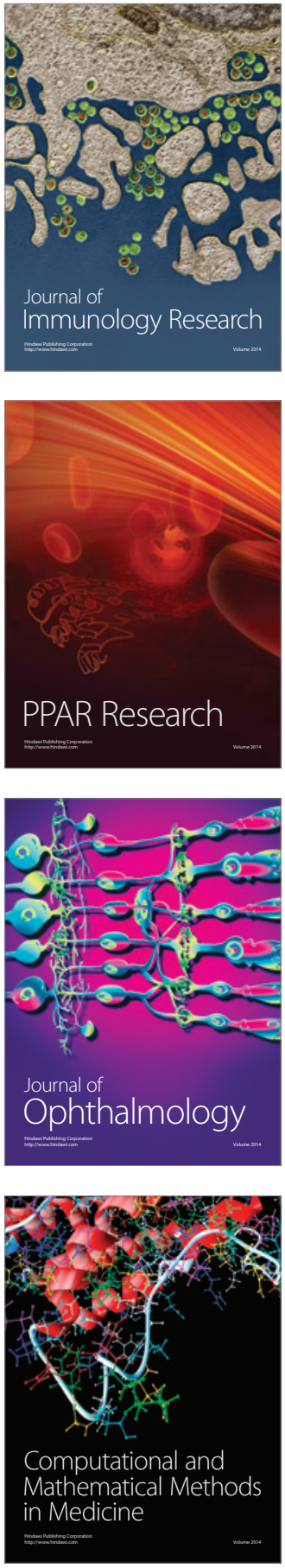

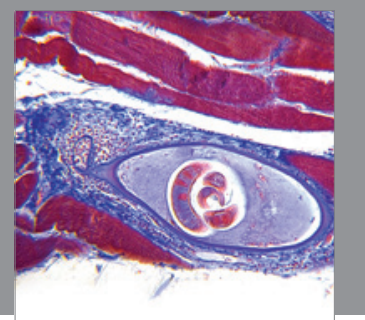

Gastroenterology

Research and Practice
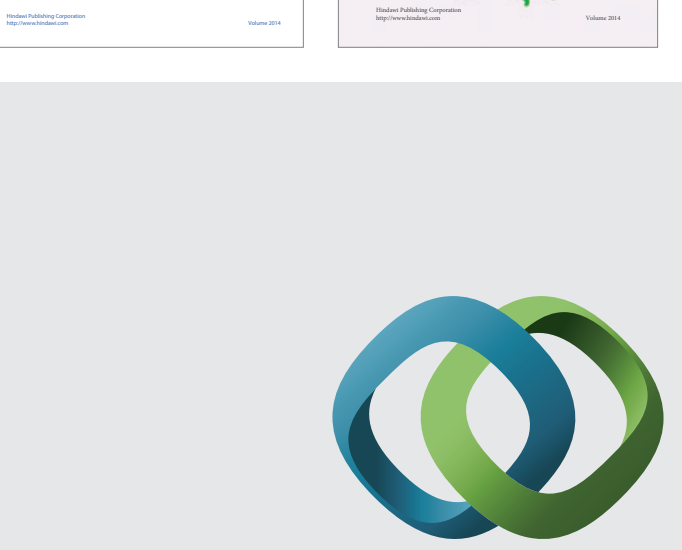

\section{Hindawi}

Submit your manuscripts at

http://www.hindawi.com
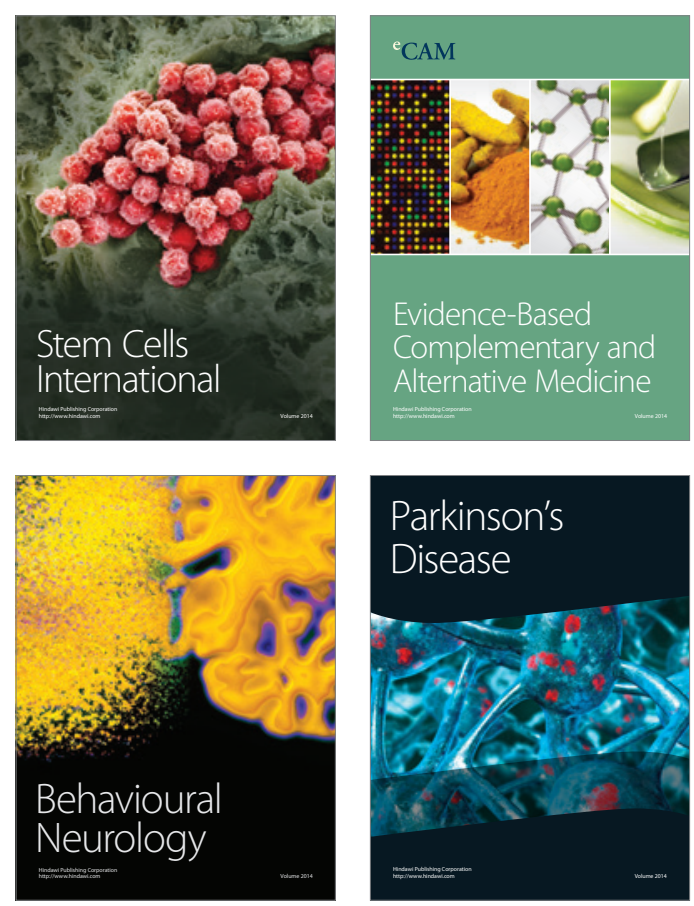

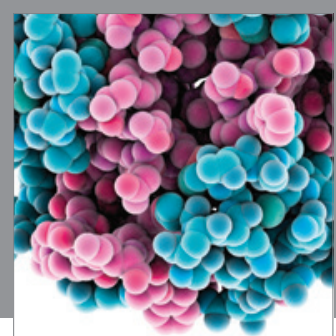

Journal of
Diabetes Research

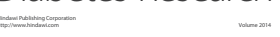

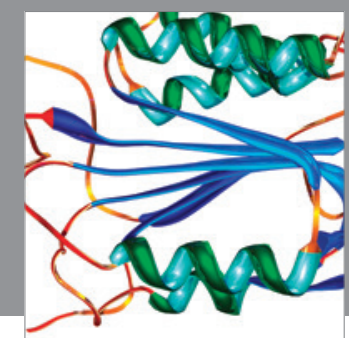

Disease Markers
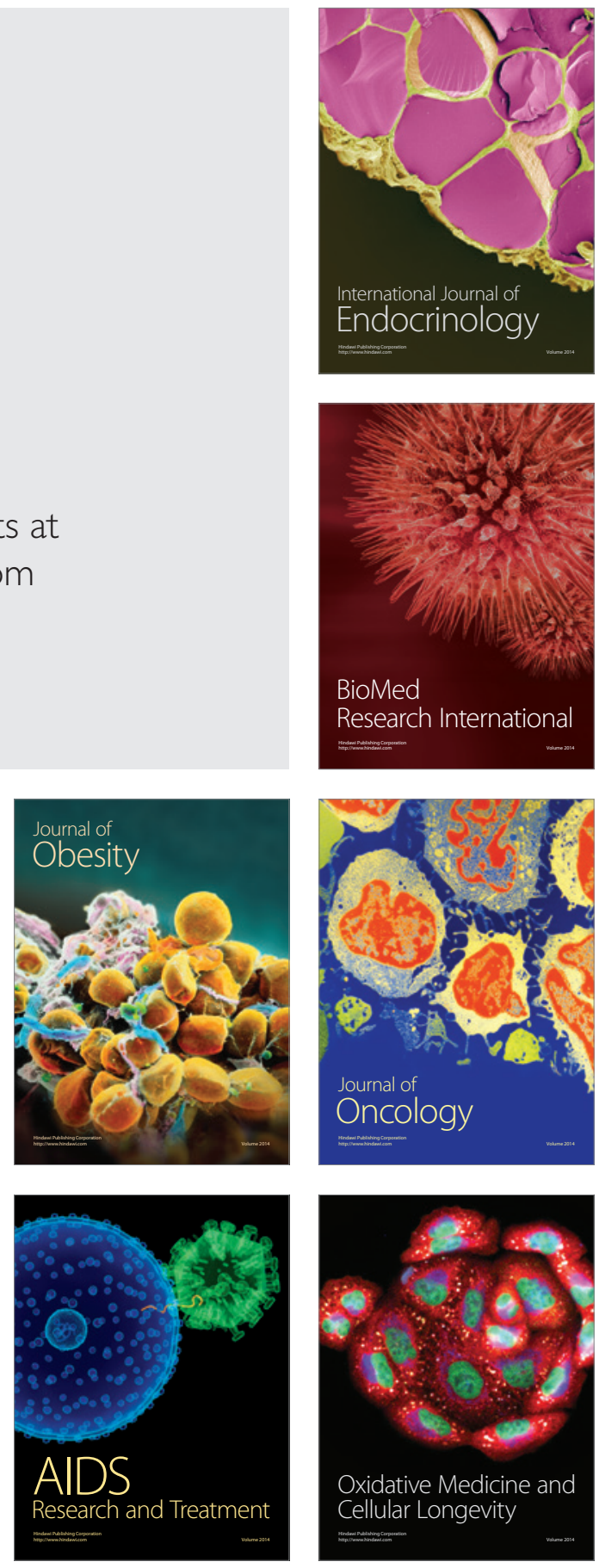